Veenendaal, B. and Brovelli, M. and Wu, L. 2016. Cloud/web mapping and geoprocessing services - Intelligently linking geoinformation. ISPRS Journal of Photogrammetry and Remote Sensing. 114: pp. 243-244.

\title{
Cloud/web mapping and geoprocessing services - intelligently linking geoinformation
}

Bert Veenendaal (Curtin University, Perth, Australia), Maria Antonia Brovelli (Politecnico di Milano, Como Campus, Italy) and Lixin Wu (China University of Mining \& Technology, Xuzhou, China)

We live in a world that is alive with information and geographies. "Everything happens somewhere" (Tosta, 2001). This reality is being exposed in the digital earth technologies providing a multidimensional, multi-temporal and multi-resolution model of the planet, based on the needs of diverse actors: from scientists to decision makers, communities and citizens (Brovelli et al, 2015). We are building up a geospatial information infrastructure updated in real time thanks to mobile, positioning and sensor observations. Users can navigate, not only through space but also through time, to access historical data and future predictions based on social and/or environmental models. But how do we find the information about certain geographic locations or localities when it is scattered in the cloud and across the web of data behind a diversity of databases, web services and hyperlinked pages? We need to be able to link geoinformation together in order to integrate it, make sense of it, and use it appropriately for managing the world and making decisions.

Linking geoinformation together is more difficult than what it first seems. There is such a host of data and information from many sources, in many forms, with many varied meanings, using many different vocabularies in many different situations and applications (Li et al., 2011). This diversity is compounded by the way the information is represented, such as, the many different spatial references and coordinate systems used (Wu et al, 2013), to the way the information is presented, such as, how web maps are communicated to a wide range of different users (Veenendaal, 2015). For example, how do we access and integrate geographic and demographic data (from a GIS database in the cloud), environmental sensor data (eg. temperature and rainfall sensor observations), hydrological spatial model services (via a geoprocessing web service) and before/after imagery to analyse the impact of flood disasters on residents' health and well-being in a particular local community?

What is required to address this is greater integration of and easy access to diverse data sets, services and workflows by users and applications. Greater automation and more intelligence is necessary to resolve differences and enable linkage and integration, including vocabularies, ontologies, analytical tools, spatial references, modelling environments and geoprocessing workflows (Figure 1). The papers in this special issue address a number of important problems needed to intelligently link geoinformation in a cloud and web services environment. In particular, they address the issues of, linking data from disparate sources into a published geoprocessing workflow, integrating and sharing multiple geo-analysis models, enhancing the user map experience of gradual content zoom and progressive transfer when accessing geoprocessing web services, and providing a user needs driven geo-analytic environment that integrates and orchestrates multiple dynamic geoprocessing web services. 


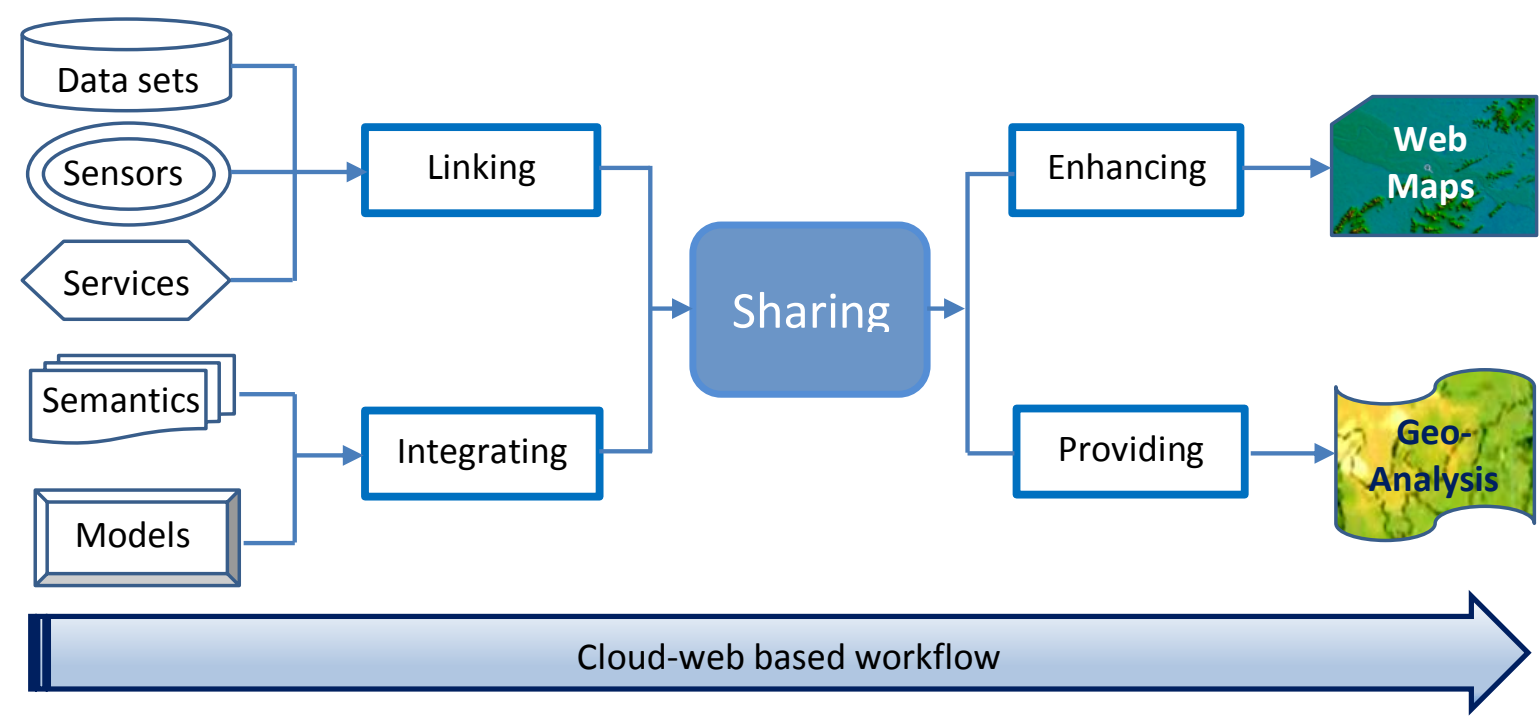

Figure 1: Workflow to intelligently link geoinformation

In order to make effective use of the myriads of data accessible on the web, it needs to be linked so that it can be appropriately integrated, shared and provide useful information to users and decisionmakers. The concept of Linked Data was coined by Berners-Lee (2006) in the context of the semantic web as an important ingredient to the web of data. Data needs to be linked so that persons and machines can find related data to be able to explore the web of data. The paper by Yue et al. (2016a) uses a Linked Data approach to link together various data resources such as sensors, observations, services, workflows and provenance, in a geoprocessing workflow to support semantic integration and discovery of geospatial information. In the context of soil moisture data access and query, they show how resources such as soil moisture sensor observations, NDVI imagery, and geospatial web services such as soil moisture derivation models, were published and chained into a geoprocessing workflow to produce a soil moisture map.

How are multiple geo-analysis models integrated and shared in such a way that providers and users can collaborate? The existing web service standards go some way to providing common interfaces, but not all aspects of model encapsulation are addressed. Yue et al. (2016b) introduces a geoanalysis model encapsulation strategy that provides interfaces for model description, execution and deployment to support the sharing, re-using and integration of such models in an open web environment. A prototype was constructed and the strategies tested using hydrology digital elevation models.

Vario-scale data structures and data underpin the gradual content zoom and progressive transfer of vector data for web maps on desktop and mobile devices. It is important to design these correctly so that users can obtain a good web map experience when accessing and zooming into web maps on a range of devices. Both the geographic context and timely transfer of data at various levels of scale needs to be maintained. The work of Huang et al. (2016) focusses on the client in a client-server web services environment. They developed data structures for a vario-scale vector representation and tested them by experimenting with them in a web client prototype. They showed that these structures could progressively update a web map with gradual content zoom. 
As geospatial web services evolve to provide geospatial data and geoprocessed information, there is an increasing need for users to influence and have control over how they explore and interact with geospatial information. This then needs to influence how geospatial web services will provide and integrate the necessary underlying data and information. Moncrieff et al. (2016) describes a framework that extends the WPS approach to enable ongoing exploratory and user driven spatial analysis across multiple attributes, datasets and spatial/temporal scales. This visual analytic environment is illustrated by a health related case study that integrates the geoprocessing of health records and demographic data.

The role of users in the Geoweb 2.0 world extends to their contribution to the generation of geoinformation. The Geoweb 2.0 paradigm means, not only augmented computational power of computers, broadband internet communication and new technologies but also, the emergence of citizen science and the importance of the crowd. Brovelli et al. (2016) deals with user generated content and user participation in GIS by means of mobile-based applications. Starting from several examples (e.g. urban monitoring and planning, tourism valorisation) based on software developed by the authors, some general conclusions about the ways and needs of user participation are drawn. The tools presented are implemented as free and open source, ensuring a high level of re-usability of the proposed solutions.

We would like to thank all contributing authors, including those whose papers were not selected for publication in this theme section. Special thanks go to the many anonymous reviewers who provided their constructive critical recommendations and suggestions. Without their contributions, this special issue would not have been published. Finally, we would like to extend our thanks to the journal editors Professor Derek Lichti and Professor Qiao Weng for their assistance and guidance.

References

Berners-Lee, Tim. 2006. Linked Data. In Design Issues, W3C, https://www.w3.org/Designlssues/LinkedData.html. (Accessed 21 January 2016)

Brovelli Maria A., Carolina Arias Munoz \& Giorgio Zamboni. 2015. From paper maps to the Digital Earth and the Internet of Places. Geodesy and Geomatics to the edge, Rendiconti Lincei, June 2015, Volume 26, Supplement 1, pp 97-103, ISSN 2037-4631.

Brovelli, Maria A., Marco Minghini \& Giorgio Zamboni. 2016. Public participation in GIS via mobile applications. This issue.

Huang, Lina, Martijn Meijers, Radan Suba \& Peter van Oosterom. 2016. Engineering Web Maps with Gradual Content Zoom Based on Streaming Vector Data. This issue.

Li, S, B Veenendaal \& S Dragićević. 2011. Advances, challenges and future directions in web-based GIS mapping services. In Li, S, Dragićević, S and Veenendaal B (2011) Advances in Web-based GIS, Mapping Services and Applications, Taylor \& Francis Group, London, ISBN 978-0-415-80483-7.

Wu Lixin, Yu Jieqing, Yang Yizhou \& Jia Yongji. 2013. Spatial big data organization, access and visualization with ESSG. International Archives of the Photogrammetry, Remote Sensing and Spatial Information Sciences, Volume XL-4/W2, p51-56. 
Moncrieff, Simon, Ulanbek Turdukulov \& Elizabeth-Kate Gulland. 2016. Integrating geo web services for a user driven exploratory analysis. This issue.

Tosta, Nancy. 2001. GIS: more than just a map. In an interview by Computerworld, 25 June, p. 60.

Veenendaal, Bert. 2015. Developing a map use model for web mapping and GIS. The International Archives of the Photogrammetry, Remote Sensing and Spatial Information Sciences, XL-4/W7, 2015, 4th ISPRS International Workshop on Web Mapping and Geoprocessing Services, 01 - 03 July, Sardinia, Italy.

Yue, Peng, Xia Guo, Mingda Zhang, Liangcun Jiang \& Xi Zhai. 2016a. Linked Data and SDI: The Case on Web Geoprocessing Workflows. This issue.

Yue, Songshan, Min Chen, Yongning Wen \& Guonian Lu. 2016b. Service-Oriented ModelEncapsulation Strategy for Sharing and Integrating Heterogeneous Geo-Analysis Models in an Open Web Environment. This issue. 